\title{
Análisis de los artículos publicados en la revista Galicia Clínica: 2008-2012
} Analysis of the articles published in Galicia Clínica journal: 2008-2012

\author{
Iria Îniguez Vázquez, María Matesanz Fernández, David Rubal Bran, Rafael Monte Secades, Ramón Rabuñal Rey \\ Servicio de Medicina Interna. Hospital Universitario Lucus Augusti. SERGAS. Lugo
}

\section{Resumen}

Objetivo: describir y analizar la actividad desarrollada en Galicia Clínica estos últimos 4 años.

Método: Estudio bibliométrico de los artículos publicados en la revista Galicia Clínica en su nueva etapa, desde octubre de 2008 hasta septiembre de 2012. Se extrajeron los siguientes datos: tipo de artículo (editorial, revisión original, caso clínico, otros), especialidad del primer autor, hospital en el que trabaja el primer autor, y subespecialidad/tema sobre el que trata el artículo. Se han excluido los resúmenes de comunicaciones a los respectivos congresos de la Sociedad Galega de Medicina Interna.

Resultados: Se han publicado 16 números ordinarios, y 3 suplementos. En total fueron 169 artículos, de los cuales 9,5\% eran editoriales, 12,4\% originales, $23,1 \%$ revisiones, $42,6 \%$ casos clínicos, y $12,4 \%$ otro tipo de artículos. Se publicaron una media de 1,3 originales y 4,5 casos clínicos por número. Los temas tratados con más frecuencia fueron gestión, 15,4\% y enfermedades infecciosas $14,2 \%$. La mayoría de los trabajos (76,9\%) han sido realizados por médicos especialistas en Medicina Interna. El centro de trabajo fue un hospital gallego en el 78,1\%. Entre 4 centros, se reúne el $51,5 \%$ de los artículos publicados. Los hospitales gallegos universitarios aportan menos trabajos que los no universitarios (56 vs 61), aunque de mayor valor intrínseco, ya que predominan los originales, mientras que en los no universitarios predominan los casos clínicos.

Conclusión: Galicia clínica ha retomado su actividad en los últimos 4 años con una elevada participación de los internistas de Galicia. Sin embargo, nuestra producción científica y el impacto de la revista pueden mejorar. Sería aconsejable desarrollar estrategias que promuevan la actividad científica de calidad y su difusión, desde el inicio de la residencia de Medicina Interna, que cuenten con la participación de instituciones públicas, hospitales, nuestros propios servicios, tutores de residentes y de cada uno de nosotros.

\section{Introducción}

La investigación constituye una parte fundamental de nuestra profesión. Es un deber para los médicos en general, y los internistas en particular, implicarse en la producción científica, compaginando la investigación con la práctica médica'. Debe fomentarse desde el inicio de la formación como médico interno, y desarrollarse a lo largo de toda la actividad profesional. Permite la obtención de nuevos conocimientos y avances en los diferentes campos de la medicina, lo cual conlleva una mejor calidad en la asistencia a nuestros pacientes.

La investigación debe culminar con la publicación. Las razones que motivan al autor a escribir un artículo científico pueden ser tanto de índole profesional (difusión del conocimiento, labor docente o requisito de una investigación) como personal (reconocimiento y estímulo individual)2. La publicación nos enriquece, personal y profesionalmente, permite dar a conocer

\section{Abstract}

Objective: to describe and analyze the activity developed in Galicia Clinica during the last 4 years.

Methods: bibliometric study of the articles published in the journal Galicia Clínica in its new stage, from October 2008 until September 2012. The following data were extracted: type of article (editorial, original article, clinical record, others), first author specialty, first author affiliation hospital and article topic. Abstracts from the congresses of the Internal Medicine Galician Society were excluded

Results: sixteen ordinary issues were published, and three supplements. There were 169 articles, of which 9,5\% were editorials, $12,4 \%$ original articles, 23,1 \% review articles, 42,6\% clinical records, and $12,4 \%$ other type of articles. In average, 1,3 original articles and 4,5 clinical records were published per issue. The more frequent topics were about management $(15,4 \%)$ and infectious diseases (14,2\%). The majority of the articles $(76,9 \%)$ were from internal medicine specialists. Articles came from Galician hospitals in $78,1 \%$; four hospitals accounted for $51,5 \%$ of the articles. Galician University Hospitals contributed with less papers than not University Hospitals (56 vs 61\%), although original articles predominated from University Hospitals.

Conclusion: Galicia Clínica has restarted its activities with a high level of participation from the internists of Galicia. Nevertheless, both our scientific production and the impact of the journal can improve. It would be advisable to develop strategies that promote high quality scientific activity and its diffusion, from the beginning of the Internal Medicine training, which the implication of public institutions, hospitals, internal medicine services, resident tutors and all of us.

nuestra actividad, mejora la reputación de nuestro centro de trabajo, favorece el intercambio de conocimientos y la comparación con otros centros, y fomenta la interrelación y la creación de nuevas líneas de trabajo conjuntas ${ }^{3.4}$.

Las revistas permiten un registro de la actividad científica, y constituyen un reflejo del funcionamiento de las instituciones investigadoras y de las personas que las conforman, pero también son el signo de la salud científica de un país o región ${ }^{5}$.

Todos estos factores han sido valorados por la Sociedad Gallega de Medicina Interna (SOGAMI) cuando se decidió retomar la publicación de la revista Galicia Clínica. Esta revista representó, en sus 65 años de vida editorial, un foro para los médicos gallegos, renovado ahora en esta nueva etapa ${ }^{6}$.

El objetivo del presente estudio es describir y analizar la actividad desarrollada en estos últimos 4 años. 


\section{Método}

Estudio bibliométrico de los artículos publicados en la revista Galicia Clínica en su nueva etapa, desde octubre de 2008 hasta septiembre de 2012. Se han revisado a través de la página web www.galiciaclinica.info, en la cual se encuentran publicados a texto completo todos los números de esta etapa. Se extrajeron los siguientes datos: tipo de artículo (editorial, revisión, original, caso clínico, otros), especialidad del primer autor, hospital en el que trabaja el primer autor, y subespecialidad/tema sobre el que trata el artículo. Se han excluido de este análisis los resúmenes de comunicaciones a los respectivos congresos de la Sociedad Galega de Medicina Interna. Los datos obtenidos se incluyeron en una tabla Excel para su tratamiento estadístico.

\section{Resultados}

En el periodo analizado se han publicado 16 números ordinarios, 4 por año, y tres suplementos extraordinarios. En total fueron 169 artículos, de los cuales 16 (9,5\%) eran editoriales, $21(12,4 \%)$ originales, 39 (23.1\%) revisiones, 72 (42,6\%) casos clínicos, y 21 (12,4\%) otro tipo de artículos (comentarios clínicos, humanidades, artículos especiales, etc.).

Se publicaron una media de 1,3 artículos originales por número. Más frecuentes han sido las revisiones, y aún más los casos clínicos (4,5 por número).

El tema tratado con más frecuencia fue el referente a aspectos de gestión, 15,4\%, tanto a nivel de editorial, como de originales y revisiones. El segundo tema en frecuencia fueron las enfermedades infecciosas $14,2 \%$, pero en este caso correspondió fundamentalmente a casos clínicos. El resto de temas se repartió de forma más o menos uniforme entre el resto de subespecialidades médicas (Tabla 1).
La mayoría de los trabajos $(76,9 \%)$ han sido realizados por médicos especialistas en Medicina Interna (Tabla 2). En cuanto al centro de trabajo del primer firmante, $132(78,1 \%)$ eran gallegos, frente a $37(21,9 \%)$ procedentes del resto de la península (Tabla 3). De estos, a destacar un 5,9\% de participación de hospitales portugueses (fundamentalmente casos clínicos) y un $16 \%$ de centros del resto de España (distribuidos por todo el territorio, pero destacando que un tercio de los mismos provienen del Hospital Clínico Universitario de Salamanca).

De los hospitales gallegos destacamos que solo 4 centros, Complexo Hospitalario Universitario de A Coruña (CHUAC), Complexo Hospitalario Universitario de Vigo (CHUVI), Complexo Hospitalario Xeral-Calde/Hospital Universitario Lucus Augusti (HULA) y Complexo Hospitalario de Ourense (CHOU) contribuyen con el 51,5\% de los artículos publicados, aportando el resto de hospitales gallegos el 27,8\%.

Los hospitales gallegos universitarios aportan menos trabajos que los no universitarios (56 vs 61), aunque en principio de mayor valor intrínseco, ya que predominan los originales, mientras que en los no universitarios predominan los casos clínicos. Ambos grupos colaboran por igual con los artículos de revisión. Sin embargo, la producción de fuera de Galicia está compuesta predominantemente por casos clínicos (Tabla 4).

\section{Discusión}

Analizamos los trabajos publicados en la revista en estos 4 años. Los resultados descritos tienen varios sesgos potenciales que podrían limitar su interpretación. El primer sesgo está en la clasificación de temas, ya que algunos textos podrían clasificarse en dos apartados (por ejemplo, un artículo sobre la actividad en una consulta de insuficiencia cardiaca podría encuadrarse tanto en gestión como en cardiología). Un segundo
Tabla 1. Distribución de los trabajos publicados según tema tratado y tipo de artículo

\begin{tabular}{|l|c|c|c|c|c|c|}
\hline & $N^{0}$ & $\%$ & Editorial & Original & $\begin{array}{c}\text { Revisión / } \\
\text { comentario } \\
\text { / otros }\end{array}$ & $\begin{array}{c}\text { Caso } \\
\text { clínico }\end{array}$ \\
\hline Gestión & 26 & 15,4 & 7 & 7 & 12 & \\
\hline Enfermedades infecciosas & 24 & 14,2 & & 3 & 3 & 18 \\
\hline Neumología & 14 & 8,3 & & & 5 & 9 \\
\hline Oncología & 13 & 7,7 & & 2 & & 11 \\
\hline Aparato digestivo & 12 & 7,1 & & & 1 & 11 \\
\hline Humanidades médicas & 11 & 6,5 & 1 & 1 & 8 & 1 \\
\hline Cardiología & 9 & 5,3 & & 1 & 7 & 1 \\
\hline Neurología & 9 & 5,3 & & 4 & 2 & 3 \\
\hline Endocrinología & 6 & 3,6 & & & 5 & 1 \\
\hline Psiquiatría & 5 & 3,0 & & 1 & 4 & \\
\hline Reumatología & 3 & 1,8 & 2 & 1 & & \\
\hline Nefrología & 2 & 1,2 & 1 & & 1 & 2 \\
\hline Otros & 35 & 20,7 & 7 & 1 & 13 & 14 \\
\hline Total & 169 & 100 & 16 & 21 & 60 & 72 \\
\hline
\end{tabular}

Tabla 2. Distribución de los trabajos publicados según la especialidad del primer firmante

\begin{tabular}{|l|c|c|}
\hline & $\mathrm{N}^{0}$ & $\%$ \\
\hline Medicina Interna & 130 & 76,9 \\
\hline Consellería de Sanidade (SERGAS) & 8 & 4,7 \\
\hline Endocrinología & 6 & 3,6 \\
\hline Cirugía & 6 & 3,6 \\
\hline Oncología & 4 & 2,4 \\
\hline Cardiología & 2 & 1,2 \\
\hline Digestivo & 2 & 1,2 \\
\hline Psiquiatría & 2 & 1,2 \\
\hline Otras & 9 & 5,2 \\
\hline Total & 169 & 100,0 \\
\hline
\end{tabular}


Tabla 3. Distribución de los trabajos publicados por hospitales y tipo de artículo

\begin{tabular}{|c|c|c|c|c|c|c|}
\hline & $\mathrm{N}^{0}$ & $\%$ & Editorial & Original & Revisión / comentario / otros & Caso clínico \\
\hline Complexo Hospitalario Universitario de A Coruña & 29 & 17,2 & 3 & 5 & 11 & 10 \\
\hline Complexo Hospitalario Universitario de Vigo & 21 & 12,4 & 3 & 5 & 8 & 5 \\
\hline $\begin{array}{l}\text { Complexo Hospitalario Xeral-Calde / Hospital } \\
\text { Universitario Lucus Augusti }\end{array}$ & 20 & 11,8 & 1 & 3 & 12 & 4 \\
\hline Complexo Hospitalario de Ourense & 17 & 10,1 & 3 & 1 & 1 & 12 \\
\hline Hospital Arquitecto Marcide & 9 & 5,3 & & & 5 & 4 \\
\hline Complexo Hospitalario Universitario de Santiago & 6 & 3,6 & 1 & 1 & 2 & 2 \\
\hline Complexo Hospitalario de Pontevedra & 4 & 2,4 & & & 1 & 3 \\
\hline Hospitales comarcales gallegos* & 11 & 6,5 & 1 & 3 & 2 & 6 \\
\hline Consellería de Sanidade (SERGAS) & 8 & 4,7 & & 2 & 7 & \\
\hline Hospitales de Portugal* & 10 & 5,9 & & & 1 & 9 \\
\hline Otros centros ${ }^{\star \star \star}$ & 34 & 16 & 4 & 1 & 8 & 17 \\
\hline
\end{tabular}

*Hospitales comarcales de 0 Barco, Salnés, Verín y Barbanza **Hospitales de Faro, A Guarda, Viana, Matosinhos y Porto

${ }^{* \star * H o s p i t a l e s ~ d e ~ B a d a j o z, ~ B a r c e l o n a, ~ B i l b a o, ~ C i u d a d ~ R e a l, ~ M a d r i d, ~ M a ́ l a g a, ~ S a l a m a n c a ~ y ~ V a l e n c i a, ~ A t e n c i o ́ n ~ p r i m a r i a ~ y ~ o t r o s ~}$

Tabla 4. Distribución de los trabajos publicados según el tipo de centro

\begin{tabular}{|c|c|c|c|c|c|}
\hline & \multicolumn{4}{|c|}{ Galicia } & \multirow{2}{*}{$\begin{array}{c}\text { Resto de España } \\
\text { y Portugal }\end{array}$} \\
\hline & Hospital universitario & Hospital no universitario & Otros centros & Total Galicia & \\
\hline Editorial & 7 & 5 & 2 & 14 & 2 \\
\hline Original & 12 & 7 & 1 & 20 & 1 \\
\hline Revisión - comentarios - otros & 20 & 20 & 12 & 52 & 8 \\
\hline Caso clínico & 17 & 29 & & 46 & 26 \\
\hline
\end{tabular}

sesgo está en la atribución del centro al primer autor, lo cual subestima el papel de otros centros en trabajos colaborativos. Por último, los editoriales y algunas revisiones son realizados por encargo de la dirección de la revista, lo cual sesga también los temas elegidos. Sin embargo, como más adelante se comenta, los resultados son razonables y en general concuerdan con otros estudios de ámbito similar, por lo cual los consideramos representativos.

Durante los 4 años que analizamos, se han publicado en la revista 169 trabajos, la mayoría de ellos realizados por internistas, y en los cuales han colaborado la práctica totalidad de los hospitales gallegos.

Sin embargo, muchos trabajos son de reducido valor intrínseco. La proporción de originales respecto a revisiones 0 casos clínicos es baja. Razones que pueden explicar esta situación son que los artículos de mayor calidad se publiquen en otras revistas de mayor impacto, o por el contrario, que sea la consecuencia de la escasa investigación realizada o de poco interés en publicarla. Parece más plausible ésta última explicación; cuando se analizan los méritos de investigación y académicos que presentaron los diferentes especialistas clínicos en la Oferta de Pública de Empleo del SERGAS en 2006, la actividad publicadora de los internistas parece ser inferior a la de otros especialistas, al menos en Galicia7. Los propios internistas están insatisfechos respecto a la actividad investigadora. Así cuando se les pregunta por las carencias y las necesidades de su servicio señalan en uno de los primeros lugares la actividad docente e investigadora ${ }^{8}$.

Asimismo, en una encuesta realizada en 2010 a los médicos internos residentes (MIR) de medicina interna en Galicia, el $78 \%$ refería no haber publicado nunca ${ }^{9}$, y esto no parece cambiar con el paso de los años ya que en la encuesta realizada en 2012 el 71,8\% tampoco había publicado, y los que sí lo habían hecho, presentaban menos de una publicación por residente ${ }^{10}$.

Solo el 12\% de los MIR ha participado en proyectos de investigación y sólo el 15\% han cursado el Diploma de estudios avanzados (paso necesario para poder realizar una tesis doctoral). Estos datos pueden extrapolarse a otras especialidades y comunidades, como Cataluña, País Vasco y Murcia ${ }^{11-13}$. Una encuesta de satisfacción realizada a los MIR de un hospital de tercer nivel acreditado para docencia, refleja que un $49 \%$ de los residentes no ha escrito ningún trabajo científico, el 50\% no ha realizado el curso de doctorado y sólo el $4 \%$ ha escrito la tesis doctoral (realizando más tesis los residentes de áreas quirúrgicas), manifestando su insatisfacción con su formación científica y las pocas facilidades para desarrollarla ${ }^{14}$.

Cuando se mide la aportación científica de la medicina interna gallega hospitalaria a congresos nacionales e internacionales de nuestra especialidad, se observa una creciente incorporación de los internistas a la investigación clínica ${ }^{15}$. Sin embargo esto no se refleja posteriormente en una publicación. Otro estudio que analiza la actividad investigadora aportada por los Internistas a Congresos Europeos en el periodo 1997- 
2003, concluye que España ocupa un lugar muy destacado en cuanto a producción congresual, sin embargo más de la mitad de los trabajos no llegan a plasmarse en publicaciones ${ }^{16}$. Lo mismo ocurre con los MIR, según la última encuesta que se ha realizado, que muestra que existe una amplia brecha entre la actividad comunicada a congresos y las publicaciones. Estas diferencias pueden explicarse porque la investigación sea de baja calidad, con lo cual no pasa el filtro de los revisores o porque por diversas circunstancias no se complete la labor investigadora con la pertinente publicación.

Este bajo interés de los internistas por la publicación se ha atribuido a múltiples factores como la actividad predominantemente clínica, una mayor presencia en hospitales comarcales respecto a los de tercer nivel y en áreas clínicas de gran presión asistencial con menos tradición publicadora, y la existencia de una menor cantidad de revistas específicamente dedicadas a la medicina interna respecto a otras especialidades, lo que supone una dificultad añadida para publicar ${ }^{7}$.

El que la mayoría de los autores sean internistas no contradice lo anterior, sino que indica la existencia de un sesgo en relación a que la revista es, junto con la web www.meiga.info, el órgano oficial de difusión de la SOGAMI ${ }^{17}$. En este sentido probablemente sería beneficioso por parte de la Sociedad implementar políticas que fomenten la participación de otros especialistas y Sociedades en la revista, lo cual enriquecería su calidad científica y permitiría crear líneas de trabajo multidisciplinares.

En el mismo sentido los autores, como es de esperar, proceden principalmente de hospitales gallegos, aunque el hecho de que una cuarta parte de los trabajos sea de fuera de Galicia está en relación probablemente no solo con la proximidad geográfica, sino con la visibilidad que aporta la publicación en abierto en la web. La futura inclusión de la revista en bases de datos bibliográficas, tema en el que se está trabajando, redundará sin duda positivamente en este aspecto.

Las discrepancias apreciadas en los temas tratados en la primera época de la revista (1929-1989) respecto a la actual reflejan las diferencias relativas a la especialidad de los autores, ya que en la primera etapa la revista estaba dirigida a todos los médicos, no solo a internistas, lo cual se refleja en la presencia de temas gineco-obstetricos, urológicos o traumatológicos ${ }^{6}$. También se marca de alguna manera la evolución de la medicina en estos años, por un lado perdiendo parte de su importancia la patología infecciosa, especialmente la tuberculosis, en relación con el progreso en la higiene pública y la aparición de los antibióticos, y por otro aumentando en los últimos años los contenidos en gestión, en relación a la práctica de una medicina mas basada en pruebas, y con economía de recursos que precisan de una planificación estricta.
Otro factor a comentar es que, a pesar de la extendida creencia de que en los hospitales universitarios es mucho más fácil la investigación, por medios, cercanía a la Universidad, etc., esto no se refleja en la revista, ya que las diferencias entre centros universitarios y no universitarios es pequeña. Tampoco se encontraron diferencias significativas en un artículo previo que valoraba la investigación de los MIR de uno y otro tipo de hospitales ${ }^{10}$. Este hecho nos permite ahondar en el tan comentado tópico de separación entre Universidad y hospital, y debería servir de reflexión para intentar establecer o mejorar los cauces de comunicación entre ambas instituciones.

En resumen, estos datos indican que nuestra producción científica es manifiestamente mejorable. Por tanto no cabe duda de la importancia de promover la producción científica desde los inicios de la formación MIR, y mantenerla a lo largo de nuestra vida profesional. Debemos desarrollar una serie de estrategias que promuevan la actividad científica de calidad y su difusión, entre otras también en la propia revista de nuestra sociedad, que cuenten con la participación de instituciones públicas, hospitales, nuestros propios servicios, tutores de residentes y de cada uno de nosotros.

\section{Bibliografía}

1. Pedreira Andrade J. El internista como investigador. En: La Medicina Interna como modelo de práctica clínica. SOGAMl 2009; p 203-8

2. Ferriols Lisart R, Ferroils Lisart $\mathrm{S}$. Escribir y publicar un artículo científico original. Ed Mayo. Madrid 2005: 5-8

3. Asenjo MA, Bertrán MJ, Guinovart C, Llach M. Análisis de la reputación de los hospitales españoles: relación con su producción cientofica en cuatro especialidades. Med Clin (Barc) 2006; 126: 768-70

4. Giménez-Gómez N, Pedrazas D, Medina E, Dalmau D et al. Formación en investigación: autopercepción de los profesionales sobre sus necesidades. Med Clin (Bar) 2009; 132(3):112-117.

5. Mena Galindo JA. ¿Por qué publicar en una revista científica? Esdrújula. Revista de filología. 2012; 1: 37-38. Disponible en http://www.losfilologos.com/esdrujula/02_2012/ Esdrujula_2-2012.pdf

6. Montes Santiago J, Rabuñal Rey R, Monte Secades R. Galicia Clínica: Un faro en la medicina gallega en años difíciles (1930-1989) Galicia Clin 2009; 70: 15-18

7. Rabuñal R, Monte R, Paz F, Bal M, López S, Arias C et al. Análisis de la actividad investigadora de los internistas gallegos a partir de los resultados de la oferta pública de empleo. An Med Interna (Madrid) 2007; 24: 365-8.

8. Estudio Socioprofesional de la Medicina Interna en España: prospectiva 2010. Madrid: Sociedad Española de Medicina Interna (SEMI), 2005

9. Meijide H, Rabuñal R, De la Iglesia F. La formación de residentes de medicina interna en Galicia: encuesta de opinión. Galicia Clin 2010; 71: 153-9.

10. Î́iguez I, Rubal D, Matesanz M, Ventura P, Rabuñal R, Monte R. Actividad investigadora de los residentes de medicina interna de Galicia. Galicia Clin 2012; 73: 7-10

11. Pujol R, Nogueras A. La formación de residentes de medicina interna. A propósito de una encuesta de opinión. Med Clin (Barc) 2002; 118: 545-50.

12. Pijoan Jl, Urkaregi A, Morán J. Evaluación por los médicos internos residentes de la formación recibida en los servicios hospitalarios: una herramienta de monitorización. Gac Sanit 2001; 15: 432-40.

13. Puerta JL, Moreno JM, Bravo S, Gutiérrez-Fuentes JA. Valoración de la investigación que se realiza en los hospitales españoles. Rev Clin Esp 2011; 211: 169-78.

14. Ríos Zambudio A, Sánchez Gascón F, González Moro L, Guerrero Fernández M. Formación investigadora durante la residencia MIR. Encuesta de satisfacción. Rev. esp. enferm. dig. 2004; 96: 695-704

15. Montes Santiago J, Pérez Álvarez R. Aportación científica de la medicina interna hospitalaria a congresos nacionales e internacionales de su especialidad. An Med Interna (Madrid) 2002; 19: 567-70

16. Montes-Santiago J, Álvarez Muñiz ML, Martínez López M, Baz Lomba A. Proyección europea de la Medicina Interna española. Rev Clin Esp. 2006; 206: 90-4.

17. Fernández R. La recuperación de nuestra historia y la ilusión de una nueva etapa. Galicia Clin 2008; 69: 4-5 